\title{
COMMENT
}

\section{Innovation and value-driven engineering}

\author{
Frank L. Douglas
}

\section{Emerging strategies to simultaneously catalyse rewardable innovation in the field of medical devices and reduce health-care costs could also be applicable in drug development.}

Gaining market access for new drugs has traditionally been based on demonstrating sufficient effectiveness and safety. However, in the past decade, a further challenge has become prominent: showing that the new drug provides sufficient value to the payer to justify reimbursement.

This increased focus on value, driven in part by the growing costs of providing improved health care to ageing populations based on newer treatments, is notable in many countries. For example, moves towards a valuebased system for health care in the United States have been proposed $^{1}$, emphasizing that the key long-term driver of success will be restructuring the way that health care is delivered, as this is where most of the value is created and most of the costs are incurred ${ }^{1}$. Achieving a value-based delivery system will require: more extensive measurement and analysis of treatment outcomes; more effective integration of the overall treatment for individual patients, organized around their needs; and reimbursement systems that align everyone's interests in improving value for patients ${ }^{1}$. A scheme based on such value-centred principles is being piloted in several hospitals in Massachusetts, USA.

The need for industry to respond effectively to such developments in health-care provision has been highlighted in a recent report by the Economist Intelligence Unit $^{2}$. In an associated survey of senior executives in the life sciences industry, $64 \%$ of the respondents described value demonstration as a significant challenge to their business ${ }^{2}$. The lack of a consensus definition of value that is applicable to all stakeholders was identified as a further challenge - an issue that has also recently been highlighted in an article in this journal on what constitutes a rewardable medical innovation ${ }^{3}$.

Efforts to overcome such challenges are being made in the field of medical devices. For example, a collaboration between academic and industry scientists (organized by the Austen BioInnovation Institute in Akron, Ohio, USA) recently introduced the concept of value-driven engineering (VdE) as a way to simultaneously reduce health-care costs while catalysing innovation in medical devices ${ }^{4}$. The approach is based on developing new products in line with several key principles: clinical utility driven by patient-centricity in demand, design, use and function; reduced complexity in product design; and cost savings and efficiency across the health-care system.
Given the many factors shared with drug research and development (R\&D), such principles could also be applicable here. For example, the VdE principles of increased clinical utility and decreased complexity for the end-user are illustrated by continual improvements in insulin administration devices for patients with diabetes, which increase the patient's ability to accurately self-inject the right amount of insulin at the right time. Another interesting example is Asthmapolis, a tool based on a GPS-enhanced inhaler that allows the patient and physician to track the time and location of medication use. This could aid more effective disease management and reduce treatment costs, in part by helping to identify environments that are likely to trigger an asthma attack.

VdE principles could also be applied in drug manufacturing, as illustrated by an approach to produce the key (but expensive) antimalarial drug artemisinin using a simple continuous-flow process ${ }^{5}$. The authors estimate that 400 small, simple and efficient photoreactors (costing $\sim$ US $\$ 130,000$ each) harnessing their approach could meet global needs for low-cost artemisinin and potentially deliver huge cost savings to health-care systems ${ }^{5}$.

Finally, the development of combination products composed of drugs and devices, as well as other technologies, is becoming more important as the field of personalized medicine matures. In such areas in particular, VdE is well suited to the needs to stimulate rewardable innovation and acknowledge the realities of cost sensitivity in health care. Furthermore, VdE principles could help to develop a consistent definition of value, as well as aid more effective collaboration between $\mathrm{R} \& \mathrm{D}$ and commercial departments in drug development.

1. Porter, M. E. A strategy for health care reform - toward a valuebased system. N. Engl. J. Med. 361, 109-112 (2009)

2. Kielstra, P. Reinventing biopharma: strategies for an evolving marketplace. The value challenge. Economist Intelligence Unit Limited (January 2012).

3. Aronson, J. K. et al. Defining rewardable innovation in drug therapy Nature Rev. Drug Discov. 11, 253-254 (2012).

4. Austen Biolnnovation Institute in Akron (ABIA). Value-driven engineering for US global competitiveness: a call for a national platform to advance value-driven engineering. ABIA website [online], http://www. abiakron.org/Data/Sites/1/pdf/abiawhitepaper6-14-11.pdf (2011).

5. Lévesque, F. \& Seeberger, P. H. Continuous-flow synthesis of the anti-malaria drug artemisinin. Angew. Chem. Int. Ed. Engl. 51, 1706-1709 (2012)

Competing interests statement

The author declares no competing financial interests. 